OPEN ACCESS

Edited by: Roberto Benelli, San Martino Hospital (IRCCS),

Italy

Reviewed by:

Anna Pasetto,

Karolinska Institutet (KI), Sweden Francesco Sabbatino,

University of Salerno, Italy

*Correspondence:

Tianhu Wang

650221@hospital.cqmu.edu.cn

${ }^{\dagger}$ These authors have contributed equally to this work

Specialty section:

This article was submitted to Cancer Immunity and Immunotherapy,

a section of the journal

Frontiers in Oncology

Received: 25 November 2020

Accepted: 19 April 2021

Published: 20 May 2021

Citation:

Luo Z, Zhang $H$, Xiao $Y$, Wang $R$, Zhang L, Huang $C$, Cao $Y$, Sun $C$,

Zhao Y, Lin H, Wu D and Wang T

(2021) Durable Response to Immunotherapy With Antiangiogenic Drug in Large-Cell Lung Carcinoma With Multiple Fulminant Postoperative Metastases: A Case Report

Front. Oncol. 11:633446. doi: 10.3389/fonc.2021.633446

\section{Durable Response to Immunotherapy With Antiangiogenic Drug in Large- Cell Lung Carcinoma With Multiple Fulminant Postoperative Metastases: A Case Report}

Zhilin Luo ${ }^{1 \dagger}$, Hong Zhang ${ }^{1 \dagger}$, Yajie Xiao ${ }^{2 \dagger}$, Rui Wang ${ }^{1}$, Liping Zhang ${ }^{1}$, Chenglu Huang ${ }^{1}$, Yu Cao ${ }^{1}$, Chao Sun ${ }^{2}$, Yongtian Zhao ${ }^{2}$, Hanqing Lin $^{2}$, Dongfang $\mathrm{Wu}^{2}$ and Tianhu Wang ${ }^{1 *}$

${ }^{1}$ Department of Thoracic Surgery, The Third Affiliated Hospital of Chongqing Medical University, Chongqing, China,

2 Department of Medicine, YuceBio Technology Co. Ltd., Shenzhen, China

Immunotherapy alone or chemo-immunotherapy has recently been recommended for treating advanced lung carcinoma in patients without driver mutations. However, the efficacy of immunotherapy and molecular mechanism in large-cell lung cancer (LCLC) remains unclear. Here, we reported a rare case of multiple fulminant postoperative body and mouth metastases in LCLC treating with combination immunotherapy. Initially, the patient was diagnosed as early stage LCLC and underwent a radical resection of the right lower lobe. Just one month later, multiple fulminant body and mouth lesions appeared in the right upper arm, right elbow, right waist, and tongue root. Meanwhile, serum neuron specific enolase (NSE) concentration dramatically increased from 12.12 to $30.14 \mathrm{ng} / \mathrm{ml}$. Immumohistochemistry findings demonstrated moderate PD-L1 expressions with tumor proportion score (TPS), while next-generation sequencing indicated moderate tumor mutational burden (TMB) levels and gene mutations in PBRM1 L1230P and TP53 L194R of both foci. Besides, loss of heterozygosity $(\mathrm{LOH})$ at human leukocyte antigen $(\mathrm{HLA})$ class I (HLA-A*02:03, HLA-B*55:02 and HLA-C*12:03) were detected in the right upper arm metastasis, which may facilitate malignant postoperative metastases in this case. Notably, this patient received combination therapy with anti-PD-1 antibody sintilimab plus anlotinib, and achieved a partial response for at least 12 months. Using an integrated computational method, the mutant peptide TEIPENDIPL derived from PBRM1 L1230P was predicted to be a specific neoantigen and could still be presented by HLA-B*40:01. This case suggests that immunotherapy plus antiangiogenic drug may provide an alternative therapeutic option for advanced LCLC patients without common gene mutations.

Keywords: large-cell lung carcinoma, multiple postoperative metastases, loss of heterozygosity at HLA, immunotherapy, antiangiogenic drugs 


\section{BACKGROUND}

According to the World Health Organization (WHO) guidelines, primary lung tumors can be classified into four major histological types: adenocarcinoma, squamous cell carcinoma, large-cell carcinoma and small-cell carcinoma $(1,2)$. Large cell lung carcinoma (LCLC) often happens in the periphery rather than in the center of the lung and can be distinguished with other lung cancers through pathological or immunohistochemical examinations $(1,2)$. At present, LCLC patients are individually treated based on divergent histological phenotypes and gene mutations (3). For example, actionable mutations in many common genes such as EGFR, ALK, KRAS, TP53 have been detected in some LCLC patients and provide the opportunities for appropriate targeted therapy accordingly (3).

Although platinum-based chemotherapy still served as the first-line standard therapy for those patients without targetable driver mutations (4), many patients have developed chemotherapy resistance and quickly achieved disease progression (5). To date, immunotherapy has brought in inspiring clinical outcomes in variety of cancer types, particularly for non-small cell lung cancer (NSCLC) (6). Also of note, immunotherapy alone or combination immunotherapy has recently been recommended for treating advanced lung carcinoma in patients without driver mutations (7). However, studies on the efficacy of immunotherapy in LCLC are very limited. Unlike other programmed cell death 1 (PD-1) inhibitors or programmed cell death-ligand 1 (PD-L1) inhibitors (nivolumab, pembrolizumab and atezolizumab) approved by America Food and Drug Administration (FDA), sintilimab has been approved in China for classical Hodgkin lymphoma $(8,9)$. Nevertheless, sintilimab alone or sintilimab plus chemotherapy has shown manageable toxicities and encouraging efficacies in several phase I-III clinical trials in NSCLC (stage IA-IIIB) patients in China (10-14).
In addition, there are still a subset of patients who cannot benefit from immunotherapies. Recent studies in cancer immunotherapy have shown that human leukocyte antigen (HLA) is of key importance for neoantigen presentations, which can activate the host immune system against pathogens and tumor cells $(15,16)$. Besides, loss of heterozygosity $(\mathrm{LOH})$ at HLA highly involves with the immune escape mechanism in tumor evolution and may predict poor responses in anti-tumor immunotherapy in NSCLC $(17,18)$. Whereas, there are few reports on $\mathrm{LOH}$ at HLA in LCLC.

To the best of our knowledge, this is the first case reporting multiple fulminant postoperative body and mouth metastases in LCLC, which might be facilitated by LOH at HLA. Moreover, this patient received combinational therapy with anti-PD-1 antibody sintilimab plus anlotinib and achieved a partial response (PR) for at least 12 months.

\section{CASE PRESENTATION}

A 68-year-old Chinese female non-smoker presented with cough, bloody sputum and slight shortness of breath for more than 2 months and was hospitalized in June 2019. She had no other medical history or family history like hypertension, diabetes, or hyperlipidemia. Computer tomography (CT) (Figure 1A) revealed only a mass in the right pulmonary lower lobe and no bronchial or lymph node lesions. Then, she underwent a radical resection of the right lower lobe on June 2019. Pathological examination showed TTF-1(-), CgA(-), Syn (-), NapsinA(-), P40(-), CD5/6(-), CD56(-), MelanA(-), SOX10(-), S100(-), HMB45(-), KI67 60\%(+), CK(+), CKL(+), CKH $(+)$. Taking together, she was initially diagnosed as stage Ib LCLC.

Unexpectedly, multiple fulminant body and mouth lesions was found in the right upper arm (Figure 1B), right elbow, right

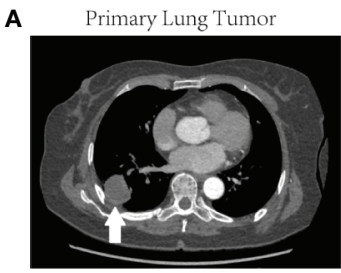

B

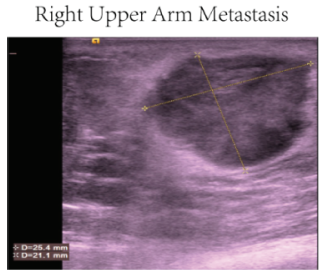

Pathological Finding

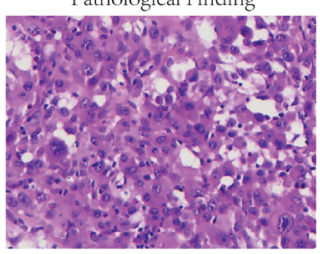

Pathological Finding

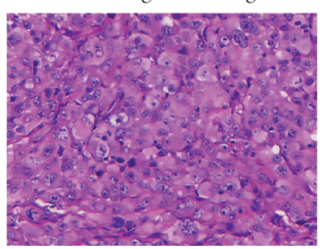

PD-L1 Expression

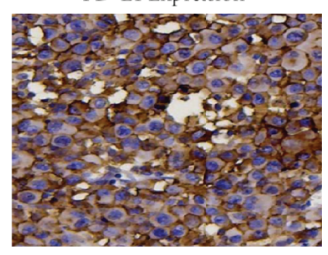

PD-L1 Expression

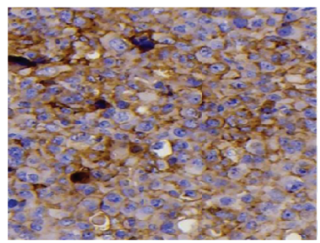

FIGURE 1 | Imaging findings, pathological results and PD-L1 expressions of primary lung tumor (A) and right upper arm metastasis (B) in this LCLC case. Initial CT scan revealed a primary lung tumor in right lower lobe, and color ultrasound scan at 1 month after operation presented a right upper arm metastasis. Pathological results of both foci were confirmed LCLC with microscope magnification at 400x. Immumohistochemistry findings demonstrated PD-L1 expression with TPS 60$70 \%$ for the primary tumor and TPS 40-49\% for right upper arm metastasis. 
waist, and tongue root at 1 month after operation. Serum neuron specific enolase (NSE) concentration dramatically increased from 12.12 to $30.14 \mathrm{ng} / \mathrm{ml}$. Further pathological examination of the surgical removal from the right upper arm presented CK (+), EMA(+), TTF-1(-), CgA(-), Syn(-), NapsinA(-), P40(-), CD5/6(-), CD56(-), Vim(+), KI67 80\% (+), CD34(+), CD31(+), ERG(+). Therefore, she was confirmed stage IVb LCLC.

Immumohistochemistry staining demonstrated PD-L1 expression with tumor proportion score (TPS) of $60-70 \%$ for the primary tumor in right lower lobe (Figure 1A) and $40-49 \%$ for the body metastasis in the right upper arm (Figure 1B) respectively. The formalin-fixed paraffin-embedded (FFPE) tumor tissue slides together with matched blood samples were sent to College of American Pathologists (CAP)-authenticated biotechnique laboratory (Yucebio, Shenzhen, China) to perform next-generation sequencing (NGS) using YuceOne ${ }^{\mathrm{TM}}$ Plus extensive targeted panel for 1,021 genes. NGS data indicated tumor mutational burden (TMB) of 2.01 muts/Mb for the primary tumor in right lower lobe and 5.36 muts/Mb for the body metastasis in the right upper arm. Driver gene mutations in PBRM1 L1230P and TP53 L194R were found both in the primary tumor of the right lower lobe (variant allele frequencies of 2.65 and $5.14 \%$ respectively) and the body metastasis of the right upper arm (variant allele frequency of $63.18 \%$ and $57.96 \%$ respectively).

Besides, the primary tumor of the right lower lobe was genotyped as $\mathrm{HLA}^{\star} \mathrm{A}^{\star} 02: 03, \mathrm{HLA}-\mathrm{A}^{\star} 11: 01, \mathrm{HLA}-\mathrm{B}^{\star} 55: 02$, HLA-B $^{\star}$ 40:01, HLA-C ${ }^{\star}$ 12:03, and HLA-C ${ }^{\star}$ 07:02 using a modified computing method (19). According to another previous study (15), LOH at HLA-A ${ }^{\star} 02: 03, \mathrm{HLA}^{*}{ }^{\star}$ 55:02 and HLA-C $C^{\star} 12: 03$ were detected in the right upper arm metastasis while no abnormality was found at HLA-A*11:01, HLA-B ${ }^{\star}$

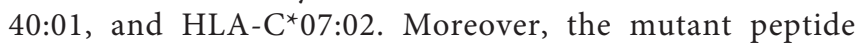
TEIPENDIPL derived from PBRM1 L1230P was predicted to be a specific neoantigen using an integrated tool TrueNeo (20) and could still be presented by HLA-B ${ }^{\star} 40: 01$ in both foci.

Considering moderate expressions of predictive biomarkers for immunotherapy (PD-L1 expression levels $<50 \%$ and TMB $<10$ muts $/ \mathrm{Mb}$ ) and fulminant metastases, this patient started chemoimmunotherapy with sintilimab $(200 \mathrm{mg})$ plus etoposide (100 $\mathrm{mg} / \mathrm{m}^{2}, \mathrm{~d} 1 \sim 3$ )-lobaplatin (50 mg/kg, d1) on July, 2019. Then, she suffered from several classical side effects of chemotherapy such as nausea, vomiting and fatigue, and refused to take any further chemotherapy. Although she developed a new lesion in left back and lymph node enlargement in left clavicle, there was no abnormity in the lung. Afterwards, she turned to another combination immunotherapy strategy of sintilimab (200 mg) plus multitargeted antiangiogenic agent anlotinib $(12 \mathrm{mg})$ in August 2019. After 1-cycle treatment, multiple body lesions shrunk. Then, serum NSE level gradually decreased to $16.63 \mathrm{ng} /$ $\mathrm{ml}$ (Figure 2A) and mouth lesion (Figure 2B) disappeared for 3cycle continuous treatment in October 2019. In addition, there were no abnormity in multiple organs (Figure 2C). Besides, all the body lesions disappeared. The only remaining body lesion in the left back largely lessened and seemed like a fibrosis or scar in physical examinations. Thus, she achieved PR according to the therapeutic effects of all body and mouth lesions.
Based on the follow-up results, she has been tolerant without any unexpected side effects with combination immunotherapy and achieved a durable PR for at least 12 months till October 2020. Written informed consent was obtained from the patient for publication of this manuscript and any accompanying images. This patient is considering further lines of combination immunotherapy.

\section{DISCUSSION}

Immunotherapy with PD-1 inhibitors or anti-cytotoxic $\mathrm{T}$ lymphocyte antigen- 4 antibodies, has emerged as a promising therapeutic strategy for patients with lung cancers. Unlike other common non-small cell lung cancers, potential molecular mechanism and therapeutic efficacy of immunotherapies in LCLC are still unclear.

Regarding moderate expression of predictive biomarkers for immunotherapy and no specific driver gene mutations, it was noteworthy that loss of HLA-A ${ }^{\star} 02: 03$, HLA $^{\star}{ }^{\star}$ 55:02 and HLA$C^{\star} 12: 03$ were detected in the right upper arm metastasis. Several recent studies have emphasized the important roles of cancerspecific neoantigens in determining cytolytic $\mathrm{T}$ cell activity and in predicting immunotherapy efficacy (21-23). Accordingly, downregulation of HLA genes or LOH at HLA may reduce antigen presentation and subsequently facilitate immune evasion $(15,18,24)$. Lately, McGranahan et al. established a software tool to align sequencing reads with known human reference genome for a patient's specific HLA type to calculate HLA LOH from 90 NSCLC patients from the TRACERx study and validated in 383 lung adenocarcinomas samples and 309 lung squamous cancer samples from TCGA database (15). They showed that HLA LOH happened more commonly in the branches instead of the trunk (15), suggesting that increased frequency of HLA LOH could enable tumor metastasis. In addition, they also demonstrated that HLA LOH may facilitate immune escape due to an active immune microenvironment (15). In consistent with their findings, we inferred that LOH at HLA-A ${ }^{\star} 02: 03$, HLA-B ${ }^{\star} 55: 02$ and HLA-C ${ }^{\star} 12: 03$ may facilitate immune evasion, and subsequently enable multiple fulminant body and mouth metastases postoperatively in this case.

To date, PD-L1 expression has been recommended to help clinicians with choosing single-agent immunotherapy (TPS $>50 \%$ ) or chemo-immunotherapy (TPS 1-49\%) (25). With the advances of NGS techniques, other biomarkers like TMB are emerged for predicting the efficacy of anti-cancer immunotherapy $(26,27)$. Besides, combination of PD-L1 expression and TMB has been presented to be related to reliable prognosis in advanced NSCLC patients following ICIs (28). Concerning moderate expressions of predictive biomarkers for immunotherapy (PD-L1 expression levels $<50 \%$ and $\mathrm{TMB}<10$ muts/Mb) and fulminant metastases, this patient started chemo-immunotherapy. After only one cycle of chemo-immunotherapy, she suffered from several classical side effects of chemotherapy such as nausea, vomiting and fatigue, and refused to take any further chemotherapy. Although she had a new lesion in left back and lymph node enlargement in left clavicle, 


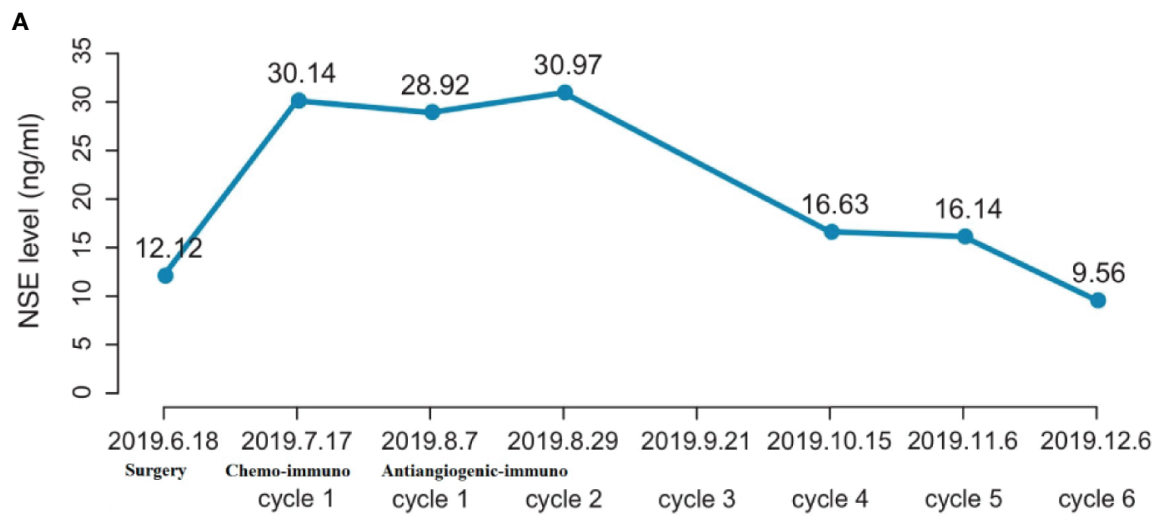

B

Before Chemo-immuno
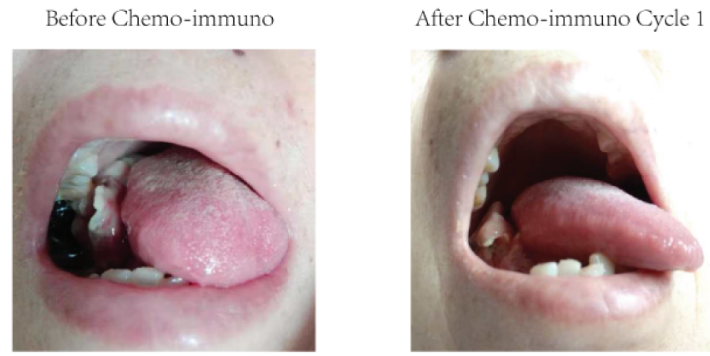

After Antiagiogenic-immuno Cycle 3

C

Lung CT after PR

Abdominal Ultrasound after PR
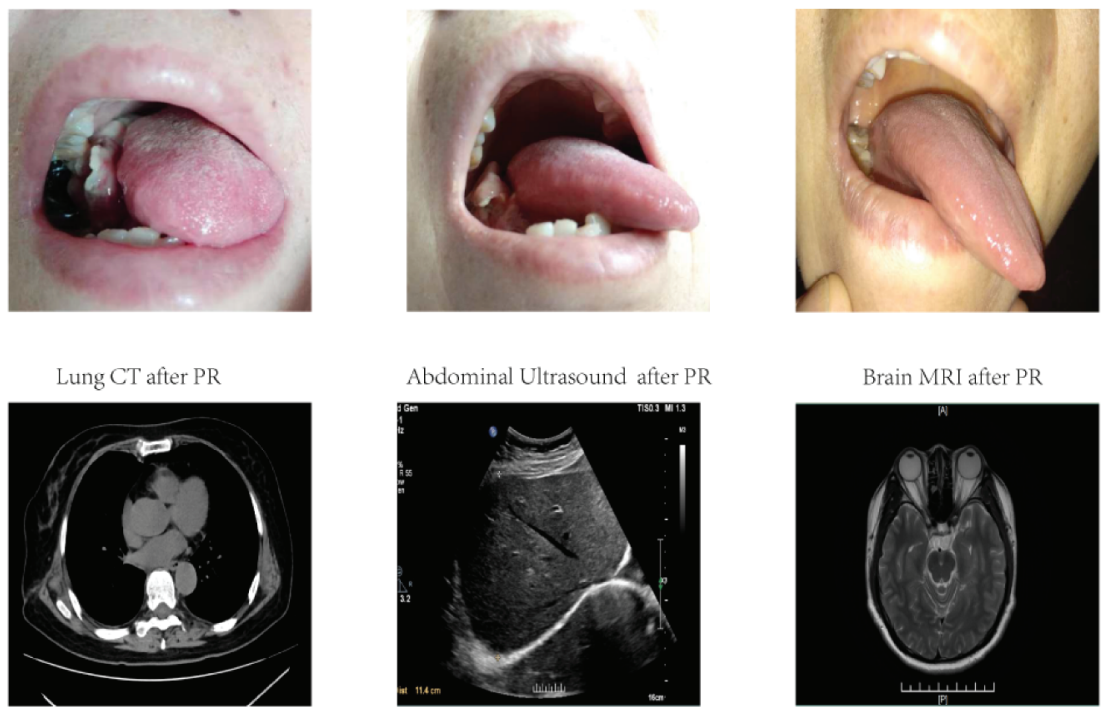

Brain MRI after PR

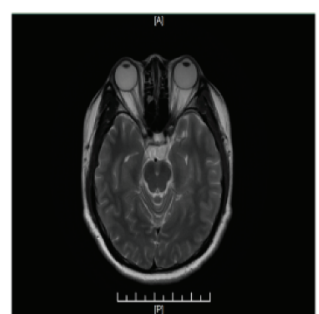

FIGURE 2 | Clinical outcomes of NSE level (A), mouth metastasis (B) and multiple organs (C) of this LCLC case. NSE level was detected before surgery or before each cycle treatment. After radical surgery, serum NSE level dramatically increased after surgery, and gradually decreased to normal levels after three cycle treatments of antiangiogenic-immunotherapy. In addition, mouth lesion appeared after surgery and disappeared after combination antiangiogenic-immunotherapy. Besides, there were no abnormity in multiple organs and PR lasted for more than 12 months.

there was no abnormality in the lung. Regarding no other common mutations like EGFR or ALK genes and patient willing, she turned to anti-PD-1 antibody sintilimab plus anlotinib and achieved a durable PR for at least 12 months. A recent phase $1 \mathrm{~b}$ study presented good efficacy and safety of sintilimab and anlotinib in untreated NSCLC patients in China without EGFR/ALK/ROS1 mutations (29). Moreover, other clinical studies also demonstrated the synergistic antitumor effects of immune checkpoint inhibitors plus antiangiogenic drugs in a variety of solid tumors $(30,31)$. On the one side, antiangiogenic therapy exhibits the potential abilities to directly or indirectly alleviate immunosuppression and neoantigen presentation (32). On the contrary, immune checkpoint inhibitors prompt tumor vessel normalization (33).

Several clinical or genetic studies have recently highlighted correlations between tumor clonal neoantigens and the recognition of tumor cells by the immune system, which also has promoted growing preclinical and clinical interests in personalized vaccines and cell therapies targeted at these tumor clonal neoantigens $(34,35)$. Using a validated computational tool (20), we predicted the mutant peptide TEIPENDIPL derived from PBRM1 L1230P to be a specific neoantigen presented by HLA-B ${ }^{\star} 40$ :01 in both foci, which may provide a potential therapeutic target for personalized therapies. Beyond peptide-MHC binding affinity prediction, this prediction method considers multiple biological steps and provides prioritization of candidate high-immunogenicity neoantigens which have been tested in vitro and compared with other prediction method (20).

This study involved some expected limitations due to the lack of biological samples. At first, we cannot do any $\mathrm{T}$ cell infiltration experiment and HLA expression analysis to 
demonstrate the definite molecular association between HLA $\mathrm{LOH}$ in body metastasis and the immune escape mechanism in tumor microenvironment. Secondly, we are not able to test $\mathrm{T}$ cell responses with the specific neoantigen predicated using IFN- $\gamma$ ELISPOT assay or other in vitro techniques. Further studies will be performed to better clarify potential molecular mechanism in LCLC and the therapeutic efficacy of immunotherapy plus antiangiogenic drugs in LCLC.

Although there are no definite guidelines for LCLC treatments, our clinical case suggests immunotherapy plus antiangiogenic drugs may provide additional therapeutic options for treating advanced LCLC patients without common gene mutations.

\section{DATA AVAILABILITY STATEMENT}

The original contributions presented in the study are included in the article/supplementary material Further inquiries can be directed to the corresponding author.

\section{REFERENCES}

1. Brambilla E, Travis W, Colby T, Corrin B, Shimosato Y. The New World Health Organization Classification of Lung Tumours. Eur Respir J (2001) 18:1059-68. doi: 10.1183/09031936.01.00275301

2. Travis W, Brambilla E, Nicholson A, Yatabe Y, Austin J, Beasley M, et al. The 2015 World Health Organization Classification of Lung Tumors: Impact of Genetic, Clinical and Radiologic Advances Since the 2004 Classification. J Thorac Oncol (2015) 10:1243-60. doi: 10.1097/ JTO.0000000000000630

3. Chan A, Chau S, Tong J, Chow C, Kwan J, Chung L, et al. The Landscape of Actionable Molecular Alterations in Immunomarker-Defined Large-Cell Carcinoma of the Lung. J Thorac Oncol (2019) 14:1213-22. doi: 10.1016/ j.jtho.2019.03.021

4. Arbour K, Riely G. Systemic Therapy for Locally Advanced and Metastatic non-Small Cell Lung Cancer: A Review. JAMA (2019) 322:764-74. doi: 10.1001/jama.2019.11058

5. Meador C, Hata A. Acquired Resistance to Targeted Therapies in NSCLC: Updates and Evolving Insights. Pharmacol Ther (2020) 210:107522. doi: 10.1016/j.pharmthera.2020.107522

6. Shen X, Zhao B. Efficacy of PD-1 or PD-L1 Inhibitors and PD-L1 Expression Status in Cancer: Meta-Analysis. BMJ (2018) 362:k3529. doi: 10.1136/ bmj.k3529

7. Yoda S, Dagogo-Jack I, Hata A. Targeting Oncogenic Drivers in Lung Cancer: Recent Progress, Current Challenges and Future Opportunities. Pharmacol Ther (2019) 193:20-30. doi: 10.1016/j.pharmthera.2018.08.007

8. Ansell S. Sintilimab: Another Effective Immune Checkpoint Inhibitor in Classical Hodgkin Lymphoma. Lancet Haematol (2019) 6:e2-3. doi: 10.1016/S2352-3026(18)30210-2

9. Hoy S. Sintilimab: First Global Approval. Drugs (2019) 79:341-6. doi: 10.1007/s40265-019-1066-Z

10. Wang J, Fei K, Jing H, Wu Z, Wu W, Zhou S, et al. Durable Blockade of PD-1 Signaling Links Preclinical Efficacy of Sintilimab to its Clinical Benefit. $m A b s$ (2019) 11:1443-51. doi: 10.1080/19420862.2019.1654303

11. Jiang H, Zheng Y, Qian J, Mao C, Xu X, Li N, et al. Efficacy and Safety of Sintilimab in Combination With Chemotherapy in Previously Untreated Advanced or Metastatic Nonsquamous or Squamous NSCLC: Two Cohorts of an Open-Label, Phase 1b Study. Cancer Immunol Immunother (2021) 70:857-68. doi: 10.1007/s00262-020-02738-x

12. Yang Y, Wang Z, Fang J, Yu Q, Han B, Cang S, et al. Efficacy and Safety of Sintilimab Plus Pemetrexed and Platinum as first-Line Treatment for Locally Advanced or Metastatic Nonsquamous NSCLC: A Randomized, Double-

\section{ETHICS STATEMENT}

Written informed consent was obtained from the individual(s) for the publication of any potentially identifiable images or data included in this article.

\section{AUTHOR CONTRIBUTIONS}

$\mathrm{ZL}, \mathrm{HZ}$, and YX acquired the data and prepared the manuscript. RW and LZ executed pathological and radiographic analyses. $\mathrm{CH}$ and $\mathrm{YC}$ interacted with the patient and did patient follow-up. CS, YZ, HL and DW performed data interpretation and revised the manuscript. TW supervised and revised the work. All authors contributed to the article and approved the submitted version.

\section{ACKNOWLEDGMENTS}

We thank the medical staff and the patient for their contribution to this study.

Blind, Phase 3 Study (Oncology pRogram by InnovENT Anti-PD-1-11). J Thorac Oncol (2020) 15:1636-46. doi: 10.1016/j.jtho.2020.09.028

13. Gao S, Li N, Gao S, Xue Q, Ying J, Wang S, et al. Neoadjuvant PD-1 Inhibitor (Sintilimab) in NSCLC. J Thorac Oncol (2020) 15:816-26. doi: 10.1016/ j.jtho.2020.01.017

14. Lam V, Forde P. Another Brick in the Wall: Sintilimab Plus Chemotherapy in Advanced Lung Cancer. J Thorac Oncol (2020) 15:1556-8. doi: 10.1016/ j.jtho.2020.08.013

15. McGranahan N, Rosenthal R, Hiley CT, Rowan AJ, Watkins TBK, Wilson GA, et al. Allele-specific HLA Loss and Immune Escape in Lung Cancer Evolution. Cell (2017) 171:1259-71.e11. doi: 10.1016/j.cell.2017.10.001

16. Sabbatino F, Liguori L, Polcaro G, Salvato I, Caramori G, Salzano F, et al. Role of Human Leukocyte Antigen System as a Predictive Biomarker for Checkpoint-Based Immunotherapy in Cancer Patients. Int J Mol Sci (2020) 21:7295. doi: 10.3390/ijms21197295

17. Anagnostou V, Smith KN, Forde PM, Niknafs N, Bhattacharya R, White J, et al. Evolution of Neoantigen Landscape During Immune Checkpoint Blockade in non-Small Cell Lung Cancer. Cancer Discov (2017) 7:264-76. doi: 10.1158/1538-7445.AM2017-NG01

18. Rosenthal R, Cadieux EL, Salgado R, Bakir MA, Moore DA, Hiley CT, et al. Neoantigen-directed Immune Escape in Lung Cancer Evolution. Nature (2019) 567:479-85. doi: 10.1038/s41586-019-1032-7

19. Yi J, Chen L, Xiao Y, Zhao Z, Su X. Investigations of Sequencing Data and Sample Type on HLA Class Ia Typing With Different Computational Tools. Briefings Bioinf (2020) bbaa143. doi: 10.1093/bib/bbaal43

20. Tang Y, Wang Y, Wang J, Li M, Peng L, Wei G, et al. TruNeo: An Integrated Pipeline Improves Personalized True Tumor Neoantigen Identification. BMC Bioinf (2020) 21:532. doi: 10.1186/s12859-020-03869-9

21. Rizvi N, Hellmann M, Snyder A, Kvistborg P, Makarov V, Havel J, et al. Cancer Immunology. Mutational Landscape Determines Sensitivity to PD-1 Blockade in non-Small Cell Lung Cancer. Science (2015) 348:124-8. doi: 10.1126/science.aaa1348

22. Rooney M, Shukla S, Wu C, Getz G, Hacohen N. Molecular and Genetic Properties of Tumors Associated With Local Immune Cytolytic Activity. Cell (2015) 160:48-61. doi: 10.1016/j.cell.2014.12.033

23. Van Allen E, Miao D, Schilling B, Shukla S, Blank C, Zimmer L, et al. Genomic Correlates of Response to CTLA-4 Blockade in Metastatic Melanoma. Science (2015) 350:207-11. doi: 10.1126/science.aad0095

24. Gettinger S, Choi J, Hastings K, Truini A, Datar I, Sowell R, et al. Impaired HLA Class I Antigen Processing and Presentation as a Mechanism of Acquired Resistance to Immune Checkpoint Inhibitors in Lung Cancer. Cancer Discov (2017) 7:1420-35. doi: 10.1158/2159-8290.CD-17-0593 
25. Mok T, Wu Y, Kudaba I, Kowalski D, Cho B, Turna H, et al. Pembrolizumab Versus Chemotherapy for Previously Untreated, PD-L1-expressing, Locally Advanced or Metastatic non-Small-Cell Lung Cancer (KEYNOTE-042): A Randomised, Open-Label, Controlled, Phase 3 Trial. Lancet (London England) (2019) 393:1819-30. doi: 10.1016/S0140-6736(18)32409-7

26. Chan T, Yarchoan M, Jaffee E, Swanton C, Quezada S, Stenzinger A, et al. Development of Tumor Mutation Burden as an Immunotherapy Biomarker: Utility for the Oncology Clinic. Ann Oncol Off J Eur Soc Med Oncol (2019) 30:44-56. doi: 10.1093/annonc/mdy495

27. Samstein R, Lee C, Shoushtari A, Hellmann M, Shen R, Janjigian Y, et al. Tumor Mutational Load Predicts Survival After Immunotherapy Across Multiple Cancer Types. Nat Genet (2019) 51:202-6. doi: 10.1038/s41588018-0312-8

28. Yu Y, Zeng D, Ou Q, Liu S, Li A, Chen Y, et al. Association of Survival and Immune-Related Biomarkers With Immunotherapy in Patients With nonSmall Cell Lung Cancer: A Meta-Analysis and Individual Patient-Level Analysis. JAMA Network Open (2019) 2:e196879. doi: 10.1001/ jamanetworkopen.2019.6879

29. Chu T, Zhong R, Zhong H, Zhang B, Zhang W, Shi C, et al. Phase Ib Study of Sintilimab Plus Anlotinib as First-Line Therapy in Patients With Advanced non-Small Cell Lung Cancer. J Thorac Oncol (2021) 16:643-52. doi: 10.1016/ j.jtho.2020.11.026

30. Powles T, Plimack E, Soulières D, Waddell T, Stus V, Gafanov R, et al. Pembrolizumab Plus Axitinib Versus Sunitinib Monotherapy as First-Line Treatment of Advanced Renal Cell Carcinoma (KEYNOTE-426): Extended Follow-Up From a Randomised, Open-Label, Phase 3 Trial. Lancet Oncol (2020) 21:1563-73. doi: 10.1016/S1470-2045(20)30436-8

31. Rini B, Plimack E, Stus V, Gafanov R, Hawkins R, Nosov D, et al. Pembrolizumab Plus Axitinib Versus Sunitinib for Advanced renal-Cell
Carcinoma. N Engl J Med (2019) 380:1116-27. doi: 10.1056/ NEJMoa1816714

32. Khan K, Kerbel R. Improving Immunotherapy Outcomes With AntiAngiogenic Treatments and Vice Versa. Nat Rev Clin Oncol (2018) 15:31024. doi: 10.1038/nrclinonc.2018.9

33. Tian L, Goldstein A, Wang H, Ching Lo H, Sun Kim I, Welte T, et al. Mutual Regulation of Tumour Vessel Normalization and Immunostimulatory Reprogramming. Nature (2017) 544:250-4. doi: 10.1038/nature21724

34. Woroniecka K, Fecci PE. Immuno-Synergy? Neoantigen Vaccines and Checkpoint Blockade in Glioblastoma. Neuro Oncol (2020) 29:1233-4. doi: 10.1093/neuonc/noaa170

35. Li L, Goedegebuure SP, Gillanders WE. Preclinical and Clinical Development of Neoantigen Vaccines. Ann Oncol (2017) 28:xii11-7. doi: 10.1093/annonc/ mdx681

Conflict of Interest: YX, CS, YZ, HL and DW were employed by YuceBio Technology Co. Ltd.

The remaining authors declare that the research was conducted in the absence of any commercial or financial relationships that could be construed as a potential conflict of interest.

Copyright (C) 2021 Luo, Zhang, Xiao, Wang, Zhang, Huang, Cao, Sun, Zhao, Lin, Wu and Wang. This is an open-access article distributed under the terms of the Creative Commons Attribution License (CC BY). The use, distribution or reproduction in other forums is permitted, provided the original author(s) and the copyright owner(s) are credited and that the original publication in this journal is cited, in accordance with accepted academic practice. No use, distribution or reproduction is permitted which does not comply with these terms. 